Thorax (1960), 15, 154.

\title{
DETECTION OF BRONCHIAL AND ARTERIAL OBSTRUCTION BY CONTINUOUS GAS ANALYSIS FROM INDIVIDUAL LOBES AND SEGMENTS OF THE LUNG
}

\author{
BY
}

\author{
P. HUGH-JONES AND J. B. WEST \\ From the Department of Medicine, Postgraduate Medical School of London, Hammersmith Hospital
}

(RECEIVEd FOR PUblication AUgUST 21, 1959)

A mass spectrometer was designed specially for clinical respiratory investigation (Fowler, 1957) and it has been in use for general tests of lung function for three years. It performs continuous automatic gas analysis, and has a gas-sampling tube of only about $\frac{1}{2} \mathrm{~mm}$. external diameter at its tip. This fine sampling tube, which is placed at the patient's lips during general tests of lung function, has now been adapted so that it can be inserted down a bronchoscope into any lobar or segmental orifice which can be seen directly.

From the results of bronchoscopic experiments on anaesthetized dogs it has been found that characteristic patterns of gas concentration changes are observed from regional bronchi during the breathing cycle and that these can be related to the ventilation, blood flow, and mechanical properties of the relevant lobe or segment. In this paper we shall first summarize these results from animal experiments and then present some examples of these characteristic patterns seen during routine diagnostic bronchoscopy in man and discuss their significance.

\section{Apparatus and Methods}

MASS SPECTROMETER.-Fowler's instrument provides a continuous analysis of the changes of gas concentration, with time, of up to four constituents of a gas mixture recorded simultaneously on a four-channel direct-writing recorder. A general description of the instrument and its performance has been published (Fowler and Hugh-Jones, 1957), and only a few facts pertinent to the present application need be given here. For regional sampling at bronchoscopy only three channels of the recorder are used to present the results of gas analysis, and these are set for recording continuously the changes in tension (partial pressure) of oxygen, carbon dioxide, and argon within the chosen lobe or segment. The argon is introduced as an inert gas into the lungs either during a single test breath, in order to measure the local ventilation within the lobe or segment, or continuously into a given regional bronchus in order to label the gas 음 coming from that particular bronchus. The fourth channel of the recorder is used either to record the total volume flow of gas expired down the bronchoscope during a test expiration or to record the changes in intra-oesophageal pressure occurring while the regional gas sampling is undertaken (see below).

The response time of the spectrometer is such that, were one gas changed instantaneously for another, $90 \%$ of full-scale deflection for the new gas would be given within one-tenth of a second. The gas flow drawn down the sampling tube by the spectrometer is approximately $20 \mathrm{ml}$. per minute. Thus, the instrument can record the very rapid variations of gas concentration which are occurring within a lobar or segmental bronchus during the breathing cycle.

Full-scale deflection on any channel can be adjusted to suit any particular requirement. In routine bronchoscopic work, the oxygen trace is set for 0-200 $\mathrm{mm} . \mathrm{Hg}$ (calibrated from atmospheric air), the carbon dioxide from $0-100 \mathrm{~mm}$. $\mathrm{Hg}$ (calibrated from a known gas mixture), and the argon set to a convenient arbitrary scale.

Bronchoscopic SAMPLING TUBES.-The sampling tube itself consists of about $4 \mathrm{ft}$. of 0.013 in. $(0.33 \mathrm{~mm}$.) bore $(0.6 \mathrm{~mm}$. outside diameter) stainless steel tubing like that used for fine hypodermic needles. This is heated electrically, to keep water vapour as a gas, the heater current being carried to within half an inch of the tip by an outer concentric steel tube of $1.2 \mathrm{~mm}$. outside diameter, electrically insulated from the finer sampling tube. This arrangement keeps the whole sampling tube reasonably rigid for ease of manipulation down the bronchoscope, but the terminal inch of the inner sampling tube itself is flexible and can be bent to any convenient angle for insertion into segmental orifices which are approximately at right angles to the main bronchi. The fine tip itself is covered by a small stainless steel wire cage which screws on to a 12 B.A. screw fixed 0.25 in. $(6 \mathrm{~mm}$.) from the end of the inner sampling tube; this cage keeps the fine projecting tip in the centre of the segmental or lobar bronchus and the whole causes little interference with the gas flow there (Fig. 1). The outer steel tube is itself covered by a layer of smooth 


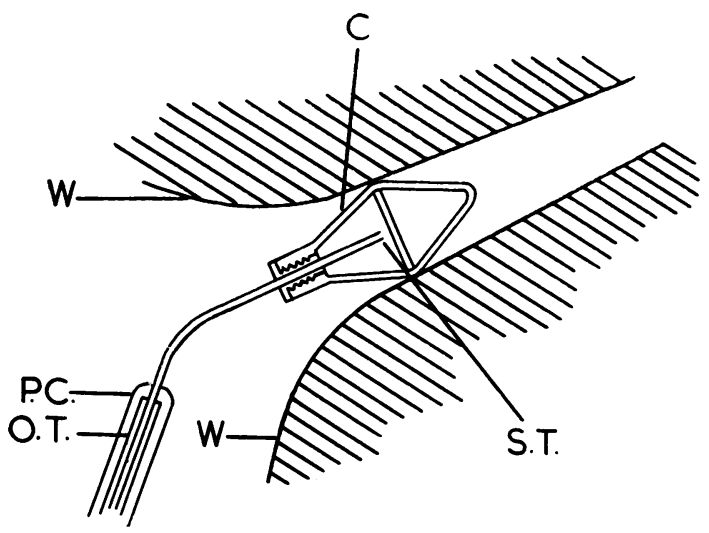

FIG. 1.-Diagram of end of mass spectrometer sampling tube in a segmental bronchus. $\mathrm{W}=$ walls of bronchus. $\mathrm{C}=$ protective stainless wire cage screwed over tip of sampling tube (S.T.), O.T. = outer stainless steel tube, P.C. = polythene covering.

plastic, so that there is adequate thermal insulation. The maximum outside diameter of the main portion of the tube is $2 \mathrm{~mm}$., that of the tip within the bronchus $0.6 \mathrm{~mm}$.

Sampling tube cages are sterilized by boiling, and the rest of the tube by immersion in a $1 \%$ solution of "sudol," a proprietary preparation of solution of cresol with soap. A two-way tap has been fitted to the spectrometer so that compressed air can be blown down the sampling tube to clear it after sterilization. Likewise, if the tube becomes blocked during the bronchoscopy, it is withdrawn from the lungs and the tap turned to clear the tube with compressed air. The tube can then be reinserted into the local bronchus and sampling resumed within less than a minute with little disturbance to the calibration of the spectrometer.

Procedure at Bronchoscopy.-A local anaesthetic is used, though additional sedation is given. Care with the anaesthetic is important both for efficiency in the procedure and for the comfort of the patient. The ideal is to have a patient so anaesthetized as to be practically unaware of the procedure but co-operative enough to take a deep breath if an argon measurement of local ventilation is required.

For most adults a $25 \mathrm{mg}$. tablet of promethazine hydrochloride is given by mouth four hours before the procedure, and, one hour before, a subcutaneous injection of a mixture of $50 \mathrm{mg}$. of promethazine hydrochloride, $100 \mathrm{mg}$. of pethidine, and $0.5 \mathrm{mg}$. of hyoscine hydrobromide. Further intravenous pethidine is used at the time of bronchoscopy, should it be necessary in nervous patients. A $2 \%$ lignocaine solution is used for local anaesthetic, with a limit of $15 \mathrm{ml}$. If intra-oesophageal pressure measurements are to be taken, a rubber balloon is inserted into the oesophagus before the bronchoscopy, when the Mackintosh laryngoscope is being used to spray the vocal cords and trachea with lignocaine.
A Negus bronchoscope is used for the bronchoscopy. This is inserted and the tree fully inspected in the usual way. The sampling tube from the spectrometer is then passed down the bronchoscope into the required lobar or segmental orifice so that the whole cage is within the relevant orifice. When this has been done, the bronchoscope itself is withdrawn into the trachea (because animal experimental work has shown that a bronchoscope in the main bronchi causes considerable interference with the normal lung ventilation) and the continuous record of the local gas concentration changes taken with the spectrometer. The bronchoscope is then reinserted lower down the bronchial tree, the sampling tube moved elsewhere, and the procedure repeated as needed.

If oesophageal pressure records are to be taken the oesophageal balloon is linked to an electro-manometer before the bronchoscope is inserted and the pressure is recorded on the fourth channel of the recorder during the bronchoscopy.

For some purposes, another fine $0.5 \mathrm{~mm}$. tube is inserted into a bronchus adjacent to that in which sampling is being done and a stream of argon fed down it. For others, a modified Negus bronchoscope is used which has an inflatable thin rubber cuff 3 in. from its distal end and has a side tube at the proximal end. When this bronchoscope is withdrawn into the trachea, after the sampling tube has been placed in the regional bronchus, the cuff can be inflated, the side orifice of the bronchoscope attached by a push-fit to a small perspex valve-box with low resistance rubber $J$-valves and a dead space of only $20 \mathrm{ml}$., and the end of the bronchoscope sealed round the sampling tube by sliding down a rubber stopper. The whole of the patient's ventilation is then through the side tube and valve-box, and on turning a tap he can take in a single breath of a gas mixture of argon (about 10\%) in air from a spirometer. The volume of the subsequent exhalation from the bronchoscope is recorded on the fourth channel of the spectrometer recorder (instead of the oesophageal pressure) by electrical integration of the signal from a wire-mesh flow-meter attached to the exit of the valve-box. When the tap is turned for this single-breath procedure during an expiration, the dead space of valve-box and tubing is automatically flushed with argon-air mixture before the test inspiration.

Since this work was started other methods of measuring air flow in the bronchial tree have been developed so that the use of the cuffed bronchoscope is often unnecessary (West, 1960). Thus, most of the tracings obtained from patients, described below, do not indicate an argon single breath.

When regional gas sampiing is completed with any of these techniques, a biopsy specimen can be taken, if required, in the usual way. Thus the whole procedure can be done during routine diagnostic bronchoscopy.

With practice it is possible, in most patients, to insert the sampling tube into the right upper lobe, middle lobe, and the individual apical and basal segments of the right lower lobe. On the left it can 
be inserted into the upper lobe and into the individual lower lobe segments. It is sometimes possible to insert it into either of the two divisions of the left upper lobe. It is fortuitous and rare to be able to insert it into any of the individual segments of the right upper or middle lobes, and it is probably nearly always impossible to enter the segments of the lingula or of the upper division of the left upper lobe.

\section{INTERPRETATION OF NORMAL PATTERNS OF Regional Gas Analysis}

A characteristic record of the changes in gas tensions locally within the bronchial tree is seen in Fig. 2, which represents those in the apical segment of the right lower lobe of a patient who was

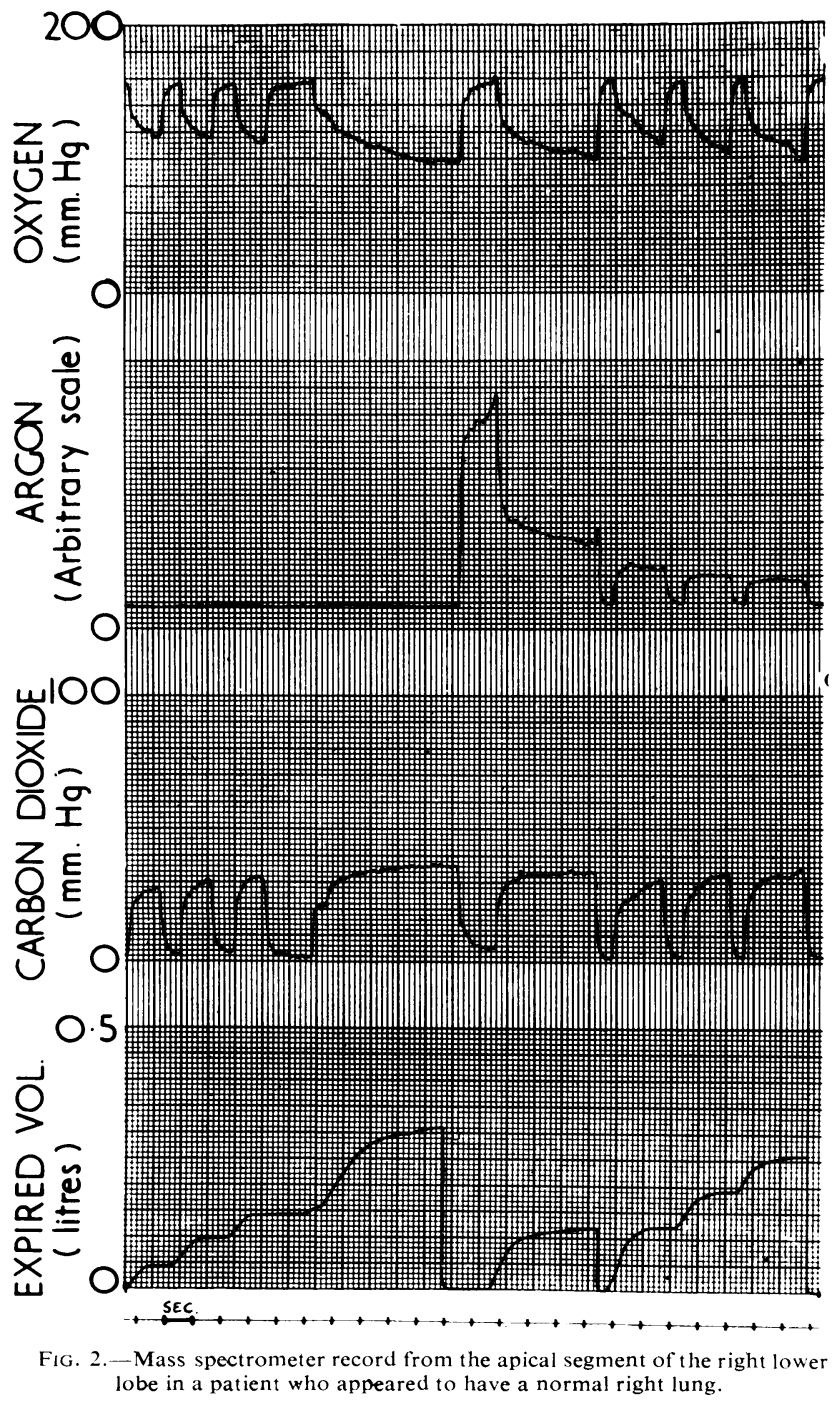

found at bronchoscopy to have an apparently normal right tree.

The tracing shows on the top three channels the record of the local changes in oxygen, argon, and carbon-dioxide tensions during breathing; and on the bottom channel the record of the total volumeen of gas expired from the cuffed bronchoscope $\overrightarrow{0}$ during each expiration.

It will be seen that the carbon-dioxide tension $\vec{\omega}$ in the segment rises to a peak during each? expiration and that the oxygen curves are almost a mirror image of the carbon-dioxide curves. $\vec{r}$ During the fourth expiration shown, the argonin was added to the air inhaled so that the $\vec{G}$ tension of argon rapidly rose in the segment, and then, during the subsequento long expiration, there is a plateau of con- $\vec{c}$ centration of this gas which is "washed out" by the three succeeding breaths of $\bar{D}$ air shown in the figure.

The interpretation of these segmental curves is perhaps easier if they are com:pared with corresponding curves repre-o senting the continuous analysis of gas passing the lips during the breathing cycle. Consider the rapid continuous $\frac{0}{\infty}$ analysis of, say, carbon dioxide at the lips (Fig. 3). On inspiration the tensiono $\overrightarrow{\overrightarrow{0}}$ of this gas falls practically to zero as there is so little in the atmosphere. On expiration there is first gas with virtually: no carbon dioxide coming from the mouth, trachea, and other parts of the anatomical dead space : then the tension. rises sharply in an S-shaped curve as $\frac{\sigma}{3}$ alveolar gas mixes with the dead-space gas, and finally there is a plateau of $\frac{0}{3}$ carbon dioxide at the alveolar tension. If the sample is taken from a segmentalo bronchus the corresponding broncho- $\rightarrow$ puimonary segment is like a miniature lung with its own dead-space gas onN expiration in the segmental bronchus." Thus the pattern for carbon dioxide will be like that at the lips, except that on inspiration the tension will not fall so sharply, as fresh gas does not immediatelyo enter the segment but is preceded by dead-space gas from the upper bronchial $\stackrel{?}{+}$ tree. Thus, although the pattern is essen-? tially the same as that at the lips, there $\frac{0}{\mathbb{D}}$

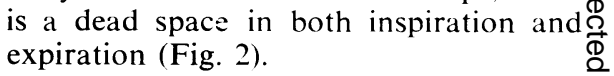

The highest point on the segmentalo argon tracing measures the argon tension 8 inhaled in a test breath of argon-airo응 


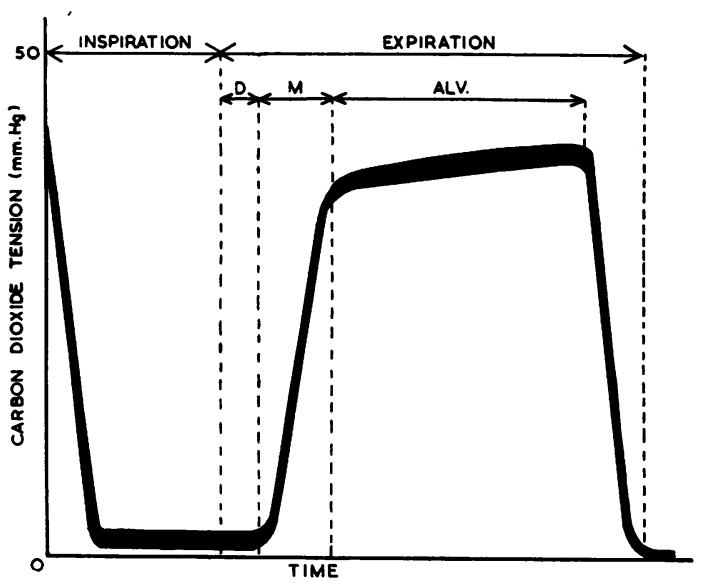

Fig. 3.-Diagram of the continuous changes in carbon dioxide concentration, recorded at the lips, when a normal subject exhales. $\mathbf{D}=\mathrm{gas}$ from upper respiratory dead space, $\mathbf{M}=$ mixed dead space and alveolar gas, alv.=alveolar plateau.

mixture. The height of the subsequent plateau during expiration depends on the dilution of the argon with the gas in the segment and is a measure of the ventilation of the segment relative to its volume.

The levels of the oxygen and carbon-dioxide plateaux during expiration are a function of the ventilation of the segment relative to its blood flow. If the perfusion is low compared with the ventilation, less carbon dioxide will be added and less oxygen extracted so that the end-expired carbon-dioxide tension will be low and the oxygen high. Conversely, if the ventilation is poor relative to blood flow, the end-expired tension of carbon dioxide will be greater and that of oxygen less. Hence there is a change in respiratory quotient, which can be related to the ventilation-perfusion ratio (West, Fowler, Hugh-Jones, and O'Donnell, 1957).

In summary, the expired argon plateau gives an indication of the segmental ventilation relative to the segmental volume and the oxygen and carbondioxide plateaux an indication of the segmental ventilation-perfusion relations. This will be seen in the abnormal tracings to be described below.

\section{Interpretation of Abnormal Patterns from ANIMAL EXPERIMENTS}

TECHNIQUE.-In order to interpret abnormal tracings found in man, dogs were bronchoscoped in circumstances where the ventilation or blood flow to the left lower lobe studied could be altered at will. Full details of the technique and the results have already been published (West and Hugh-Jones, 1959) and will only be summarized here in order to make the human tracings intelligible. The animals were anaesthetized by intravenous pentobarbitone, a tracheal cannula inserted and, under positive pressure ventilation, the chest was opened and screw clamps put round the bronchus and branch of the pulmonary artery going to the lobe. A perspex respirator was then placed over the dog with both the tracheal cannula and cables controlling the clamps led outside (Fig. 4). The mass spectrometer sampling tube was then introduced into the lobe, down a bronchoscope, as in the human technique, and the bronchoscope withdrawn into the trachea. With this arrangement, partial obstruction to the ventilation of the lobe or obstruction to the blood supply could be produced at will and the effects studied. It was found that repeatable characteristic changes in the normal lobar pattern of gas-concentration tracing were produced which can be read like an electrocardiographic pattern (Fig. 5).

Obstruction to Blood Flow. - When the arterial supply to the lobe was completely occluded, leaving its ventilation normal (Fig. 5, ii), the expired alveolar $\mathrm{CO}_{2}$ tension falls and the oxygen tension rises since little $\mathrm{CO}_{2}$ is added or oxygen extracted. The argon tracing, determined by ventilation, remains unaltered. A characteristic sharp peak, which we have called a " pip," marked $B$ in the diagram, appears at the end of the alveolar plateaux of $\mathrm{CO}_{2}$ and $\mathrm{O}_{2}$. This pip actually starts at the beginning of inspiration and arises because gas filling the upper bronchial tree (which was exhaled by the rest of the lung and has the normal oxygen and carbon-dioxide tensions of expired gas) is inhaled into the lobe past the sampling tube before fresh air reaches it.

Partial Obstruction to Ventilation.-When the blood flow was normal, but the bronchial clamp was partially screwed up so that the ventilation of the lobe was grossly reduced, the lobe was seen to ventilate out of phase with the rest of the lung because of its delayed inspiration and expiration. The characteristic pattern shown in Fig. 5, iii, was observed. The end-expired argon tension is reduced, because of the reduced ventilation. Secondly, the end-expired $\mathrm{CO}_{2}$ tension is slightly higher and the tension of oxygen slightly lower than normal because of the reduced ventilation-perfusion ratio and the slopes of the alveolar plateaux of all three gases are steeper. But most important is a small pip, marked $\mathbf{A}$ in the tracing at the start of the alveolar plateaux of $\mathrm{CO}_{2}$ and $\mathrm{O}_{2}$. This arises because there is a small time period (P, Fig. 5) when, because of the delayed inspiration along the obstructed bronchus, the lobe is still inspiring while the rest of the lung is starting to expire. Thus gas is actually " shunted" from adjacent lobes past the tip of the 


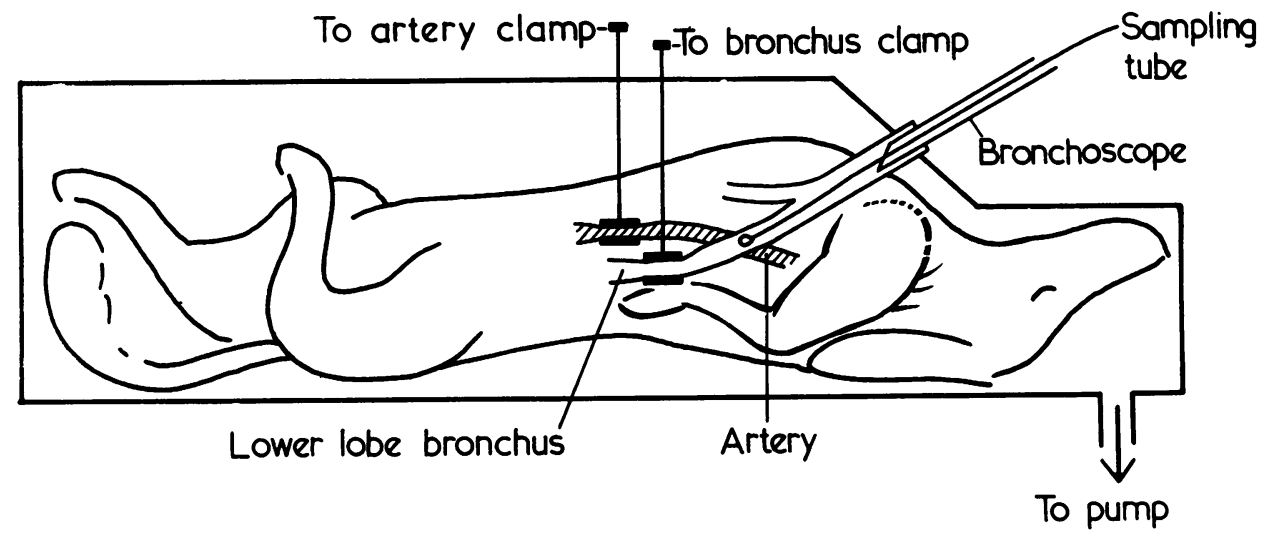

FIG. 4.-Diagram of the experimental technique in dogs for finding the effects of alteration in the ventilation and the blood flow on the pattern of gas-concentration changes within a lobe.

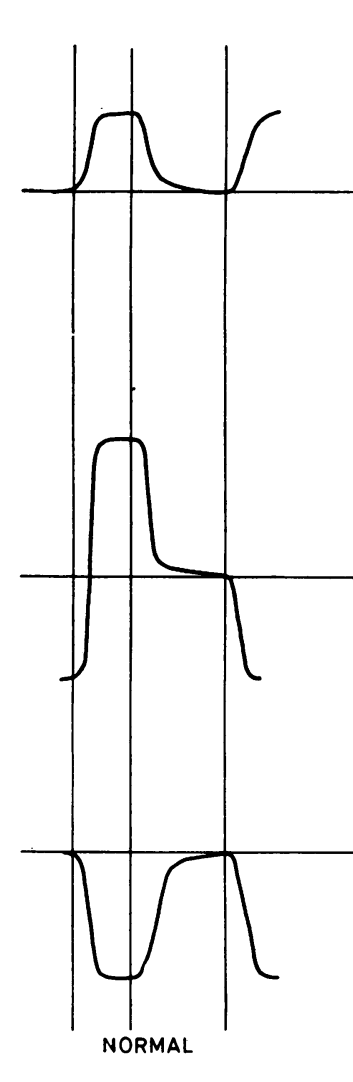

(i)

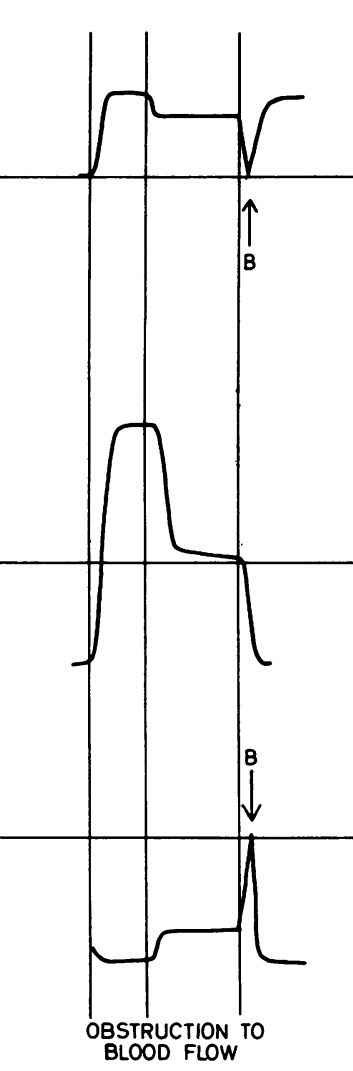

(ii)

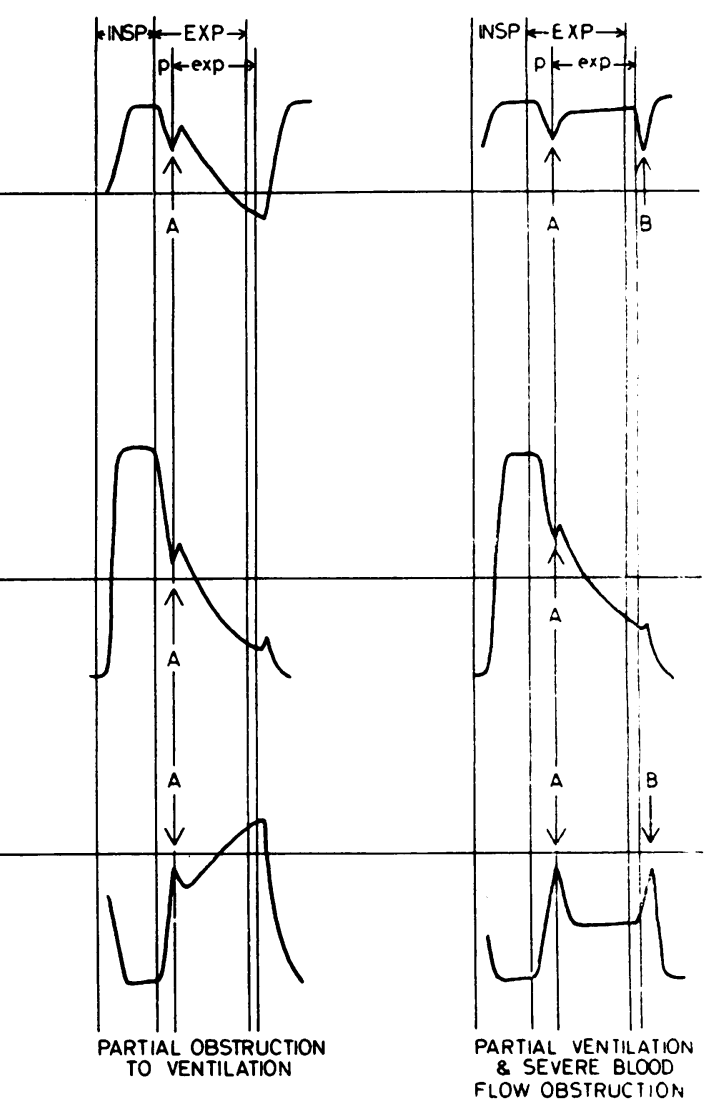

(iii) (iv)

Fig. 5.-Diagram of the effects of partial obstruction to vantilation and extreme obstruction to blood flow on the gas-concentration patterns within a regional bronchus. INSP, EXP $=$ duration of inspiration and expiration, respectively, for the whole lung. Exp $=$ duration of $\Omega$ expiration in the obstructed lobe. $P=$ time of " pendelluft" when the obstructed lobe is still inhaling but the rest of the lung exhaling. $O$ "A " and "B" pips in same tracings explained in text. 
sampling tube before the obstructed lobe itself starts to expire ("pendelluft" phenomenon). In so far as the expired gas from neighbouring lobes has a similar composition to that from the obstructed lobe, the A pip may not be very obvious.

Mixed Obstruction.-When marked arterial obstruction was produced concurrently with partial obstruction to ventilation, the pattern shown in Fig. 5 (iv) was seen. Here the argon plateau again reflects the reduced ventilation, the low $\mathrm{CO}_{2}$ and high oxygen alveolar levels reflect the lack of blood flow, and the B pip is again apparent. The A pip is now more obvious because the composition of the "pendelluft" gas differs widely from that of the alveolar gas exhaled from the experimental lobe whose blood supply is obstructed.

\section{Abnormal Patterns at Bronchoscopy IN MAN}

Blood Flow Obstruction. - An example is shown (Fig. 6) from a patient who had had extensive old tuberculous disease of the right lung. The sampling tube was sited in the right lower lobe at the level of the orifice of the medial basal segment. Comparison of this pattern with the virtually normal pattern from the left lower lobe suggested that the right lower lobe was being ventilated, but its blood supply had been grossly reduced by disease. The lobar alveolar carbon-dioxide tension was much lower and the oxygen much higher than that from the rest of the lung, so there is the characteristic $B$ pip at the start of inspiration as the diseased lobe draws in dead-space gas with a higher carbon-

Fig. 6.-Patterns obtained on the oxygen and carbon dioxide tracings from the right lower lobe of a patient with old tuberculous disease, which are typical of blood-flow diminution compared with ventilation. Time: 1 large division $=1$ second.

Fig. 7.-Comparison of a tracing from the right middle lobe bronchus and with one from the apical segment of the right lower lobe in a patient with an apparently normal right lung, showing a pattern characteristic of relative diminution in the blood flow to the right middle lobe. The effects are thought to depend on body position. Time: 1 large division $=2$ seconds.

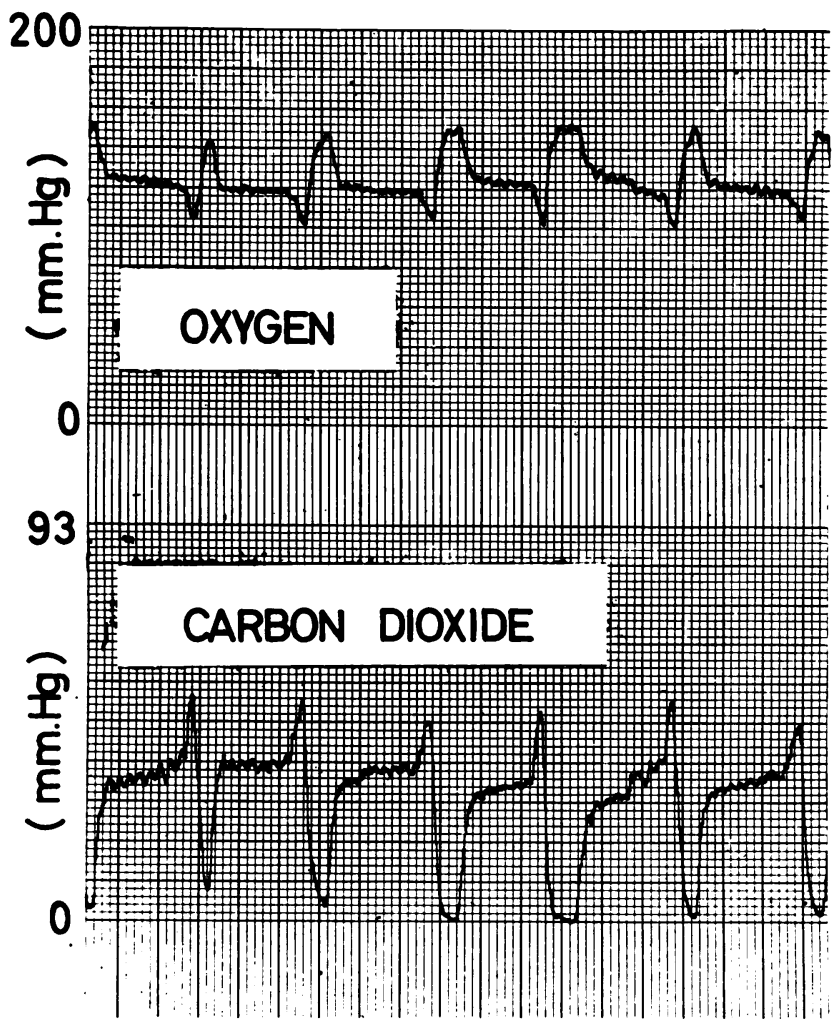

FIG. 6
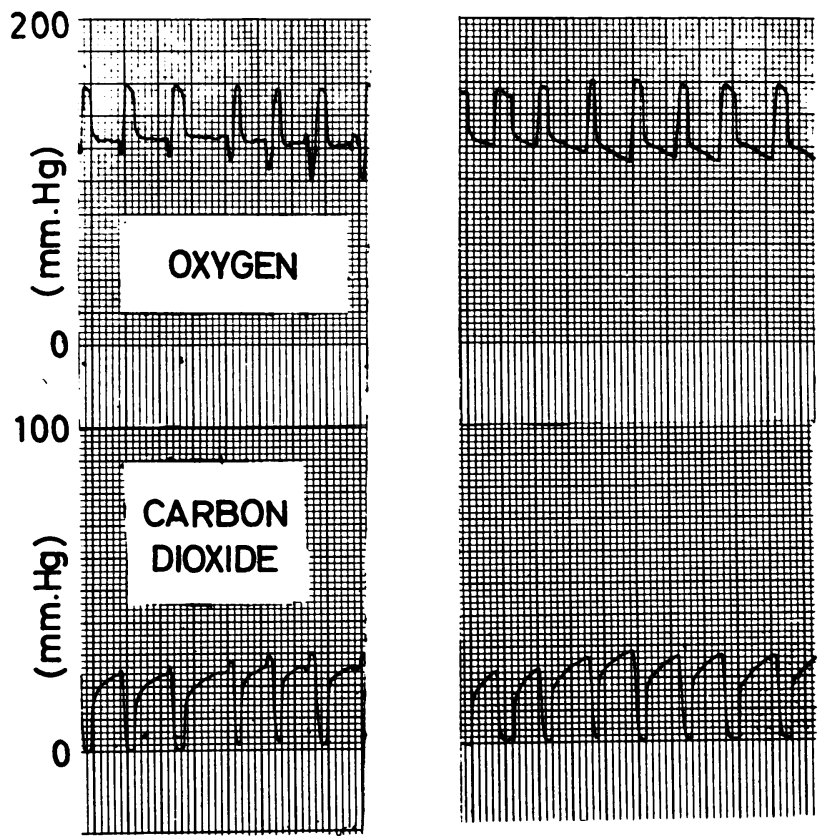

RIGHT MIDDLE LOBE FIG. 7
APICAL SEGMENT, RIGHT LOWER LOBE 


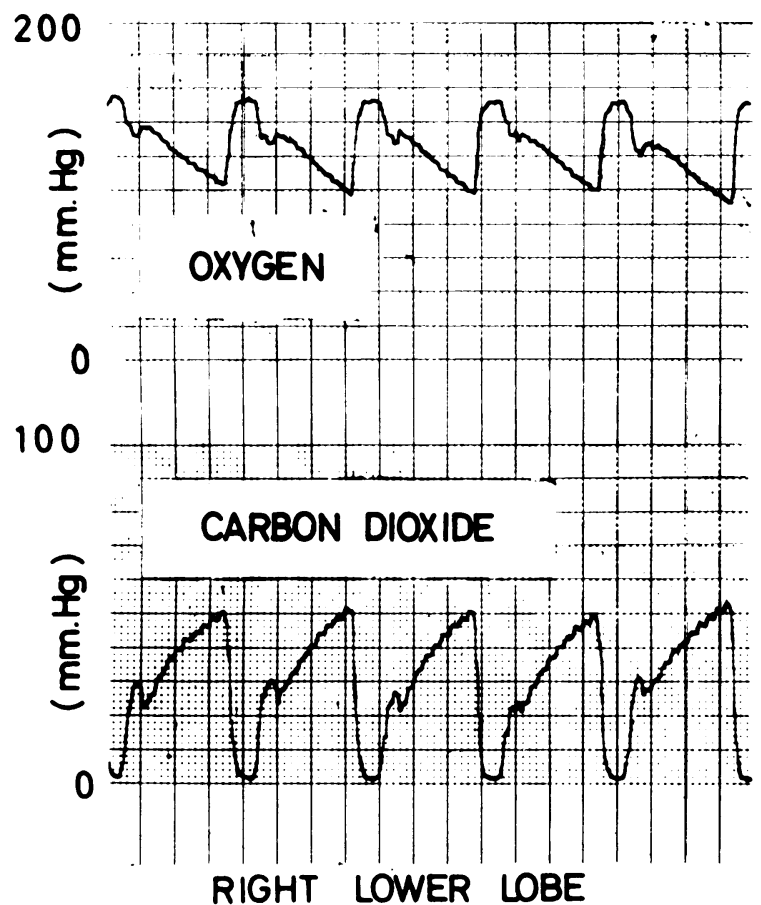

200

100

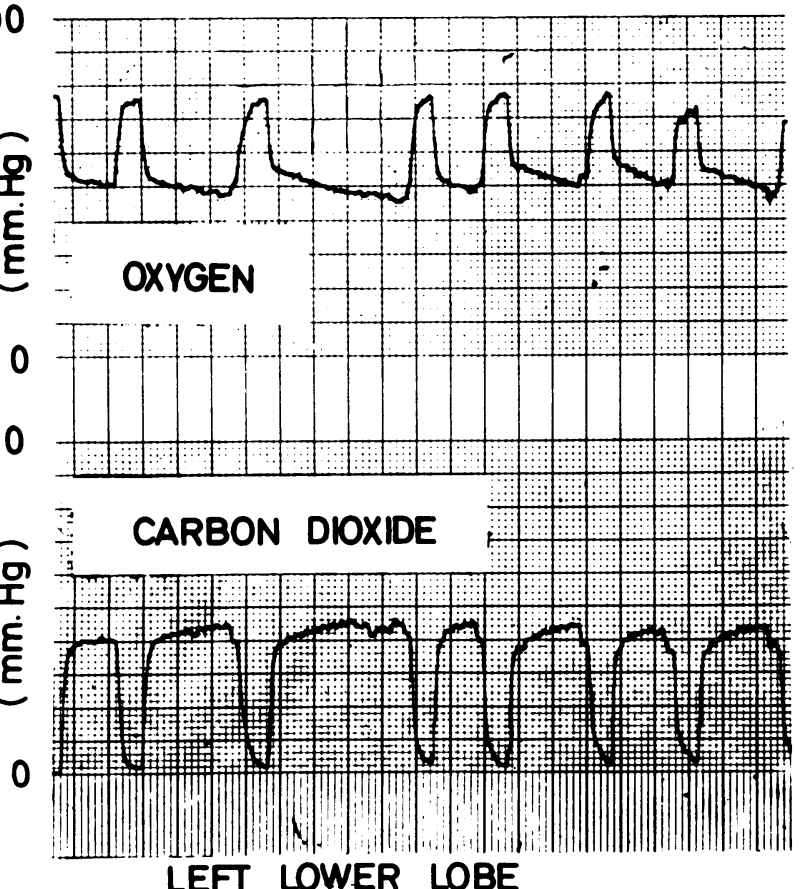

FIG. 8.-Mass spectrometer tracings from a patient whose right lower lobe was abnormally radiotranslucent compared with the left. The right lower lobe patterns are typical of those seen with partial obstruction to ventilation. Time: 1 large division $=1$ second. dioxide and lower oxygen content than its own before getting fresh air.

Sometimes this pattern of reduced blood supply with normal ventilation can be $\stackrel{\mathbb{\Phi}}{\circ}$ produced by the effects of body position $\nRightarrow$ and is not of pathological significance. This positional effect is most often seen. in the right middle lobe bronchus when $\overrightarrow{\vec{\omega}}$ the patient is bronchoscoped in the usual $\stackrel{\omega}{\omega}$ position lying on his back. Then the right $\underset{\vec{x}}{\vec{x}}$ middle lobe is uppermost and presumably has a reduced blood flow relative to its $\mathrm{G}$ ventilation compared with, say, the dependent apical lower segment (Fig. 7). G A similar excessive ventilation relative to 응 perfusion has been reported from the right upper lobe compared with the right $\subseteq$ lower in the upright posture (Mattson $\frac{5}{5}$ and Carlens, 1955) and in the uppermost lung when a patient lies on his side $\mathscr{O}$ (Svanberg, 1957; Miller, Fowler, and . Helmholz, 1956). This "middle lobe" pattern, similar to that produced by pathological obstruction to lobar or segmental blood flow, is not a constant $ٌ$ finding. It was present to a certain $\stackrel{\unrhd}{\varrho}$ extent in about half of a series of $12 \overrightarrow{\overrightarrow{\vec{O}}}$ patients bronchoscoped successively who 을 had no obvious disease of the right middle lobe.

The positional explanation of this apparently abnormal pattern in the right middle lobe has not yet been tested by turning the patient over. Till now all the $\frac{\text { O }}{3}$ regional mass spectrometer tracings have been done incidental to routine diagnostic bronchoscopy when only a short time was available.

Partial Obstruction to Ventilation. $\frac{\text { D }}{2}$ -This pattern is observed in pathological conditions, such as a tumour, causing $\widetilde{N}$ partial obstruction to the gas flow in and $N$ out of a regional bronchus, when the N time constant of the obstructed lobe is $\sigma$ prolonged compared with that of the neighbouring lobes and the ventilation is diminished relative to the blood flow.

An example is shown in Fig 8 from 0 the right lower lobe of a patient with unilateral transradiancy of the lower half $\stackrel{\Phi}{\square}$ of the right side of the chest (Macleod's $\mathbb{\mathbb { D }}$ (1954) syndrome). The pattern is contrasted with the normal pattern observed in the left lower lobe. It is a goodo 
example of this pattern and shows both the $\mathbf{A}$ pip caused by "pendelluft" gas and the steep alveolar plateaux. Other evidence in this patient showed that both the blood flow and ventilation of the right lower and middle lobes were reduced compared with the rest of the lung, but, since the ventilation appeared to be less than the blood flow, the pattern of bronchial obstruction rather than that of "mixed" obstruction was obtained. The latter requires partial ventilatory obstruction with a disproportionately great reduction in blood flow (as in the conditions of the animal experiment).

If the obstruction to ventilation is very severe, it may be virtually complete on shallow breathing, in which case no fresh air enters the obstructed bronchus, and the tracing only records variations in dead-space gas with the oxygen tension never rising to atmospheric and the carbon dioxide never falling to zero. But on deep, slow breathing, the pattern of partial bronchial obstruction appears. An example is shown in Fig. 9 from a patient with an inflammatory lesion of the right middle lobe.
At bronchoscopy the bronchus was seen to be grossly narrowed.

Mixed Obstruction Pattern.-As we have seen, this pattern is rare, for it demands a lobe or segment with some bronchial obstruction and even greater reduction of blood flow. We have only found two examples of it in over 50 routine bronchoscopies in which gas sampling was undertaken. One is shown in Fig. 10 from another patient with Macleod's syndrome affecting the right middle and lower lobes. The "dog-eared" appearance of the A and B pips in the tracing from the right middle lobe contrasts with the normal tracing from the right upper lobe. From other evidence, using the short-lived radioactive isotope oxygen-15 (Dyson, Hugh-Jones, Newbery, Sinclair, and West, 1959), this patient certainly had reduced ventilation, with delayed emptying and filling of that lobe, and a reduced blood supply. This would account for the A pip and ventilatory pattern; the position could contribute to the excessive reduction of blood flow.

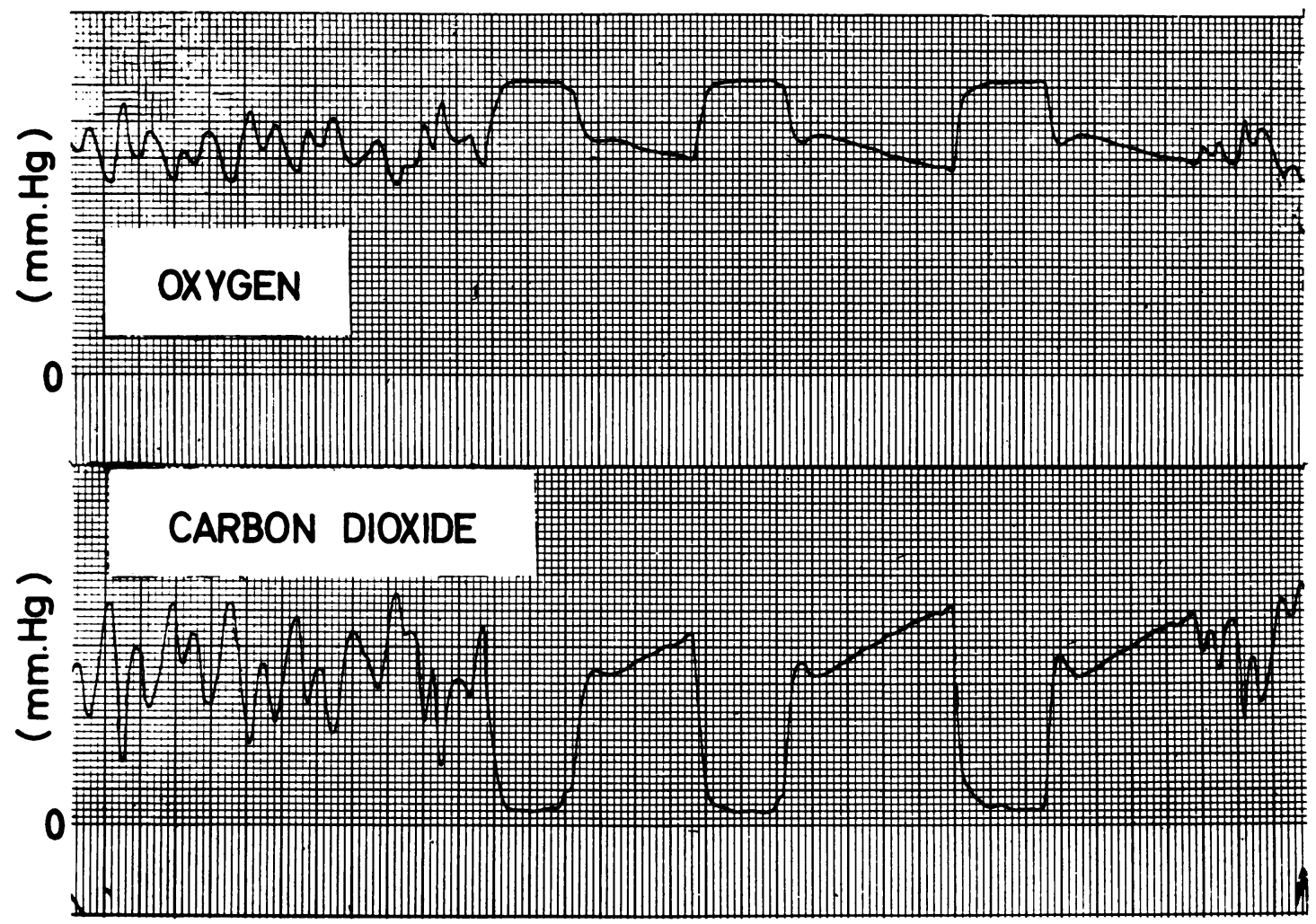

FIG. 9.- Mass spectrometer tracings from right middle lobe which had inflammatory obstruction. On shallow breathing there is no true ventilation of the lobe; dead-space gas only goes in and out past the sampling tube. On deep breathing patterns typical of partial ventilating obstruction are seen. Time: 1 large division $=1$ second. 


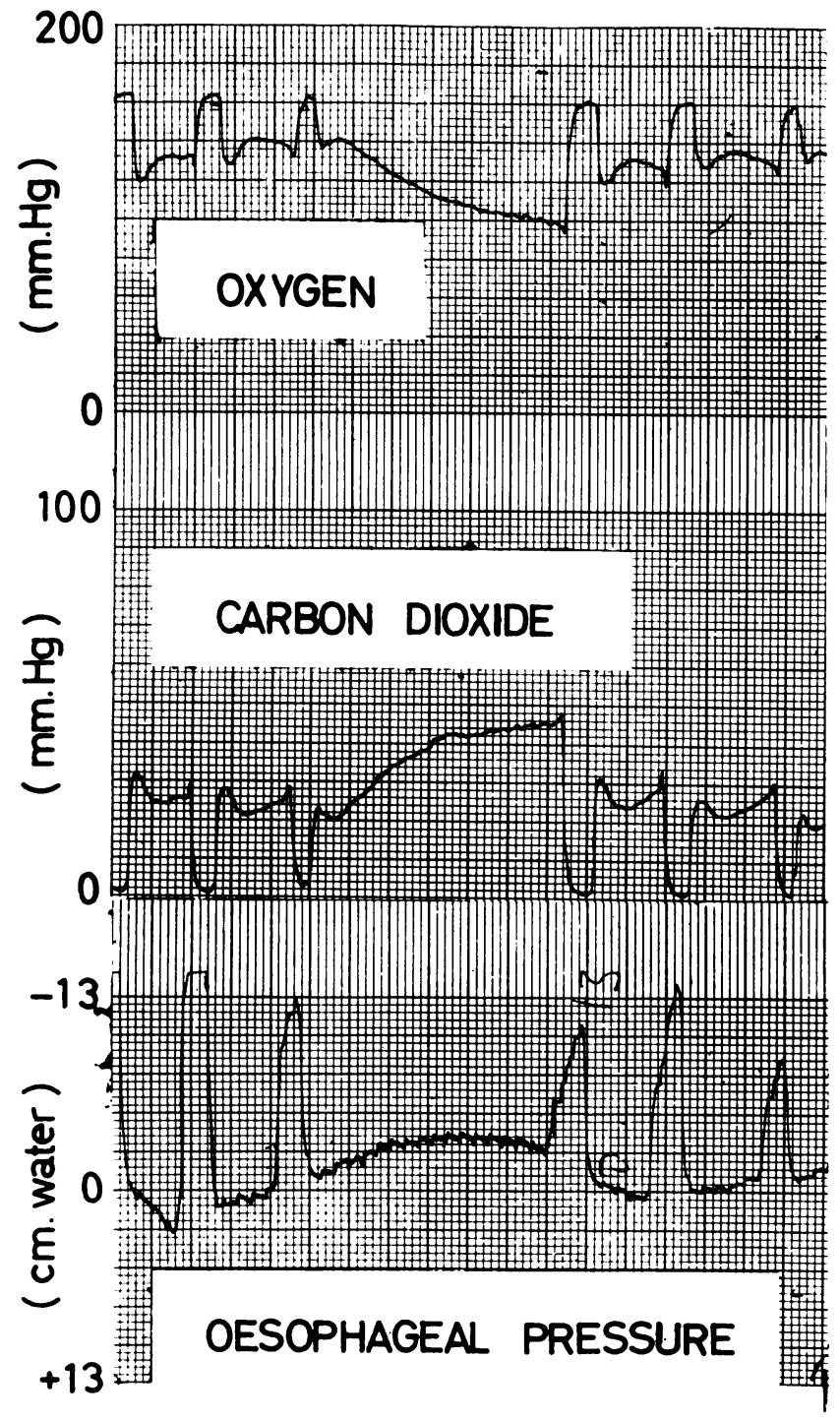

RIGHT MIDDLE LOBE

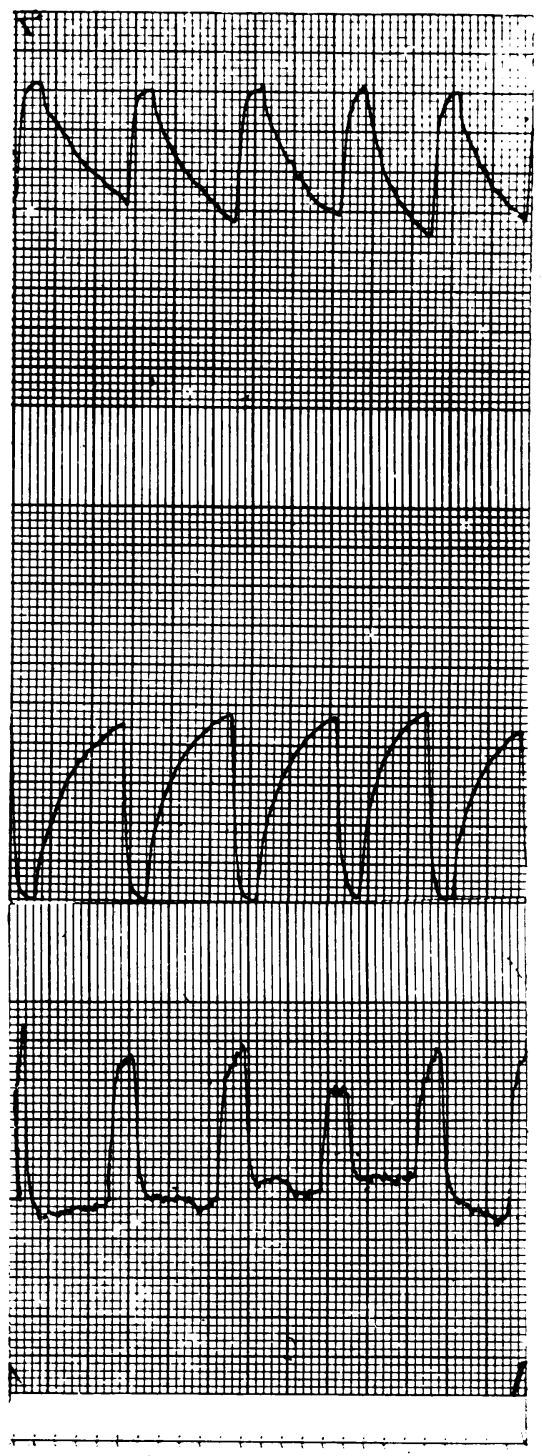

RIGHT UPPER LOBE

FIG. 10.-Tracings from a patient with the changes of Macleod's syndrome confined to the right lower and middle lobes. That from the right $\frac{\bar{\Phi}}{\Phi}$ middle lobe shows the pattern characteristic of some b:onchial obstruction with even greater blood-flow obstruction. It is contrasted $\stackrel{S}{\rightarrow}$ with the normal patterns from the right upper lobe. The $A$ and $B$ pips are shown on 4 breaths. The expiration in the centre of the tracing lasted 12 seconds and the rise in $\mathrm{CO}_{2}$ and fall in $\mathrm{O}_{2}$ tensions were then sufficient to obscure the $\mathrm{B}$ pip. Time: 1 large square $T^{2}$ 2 seconds. 


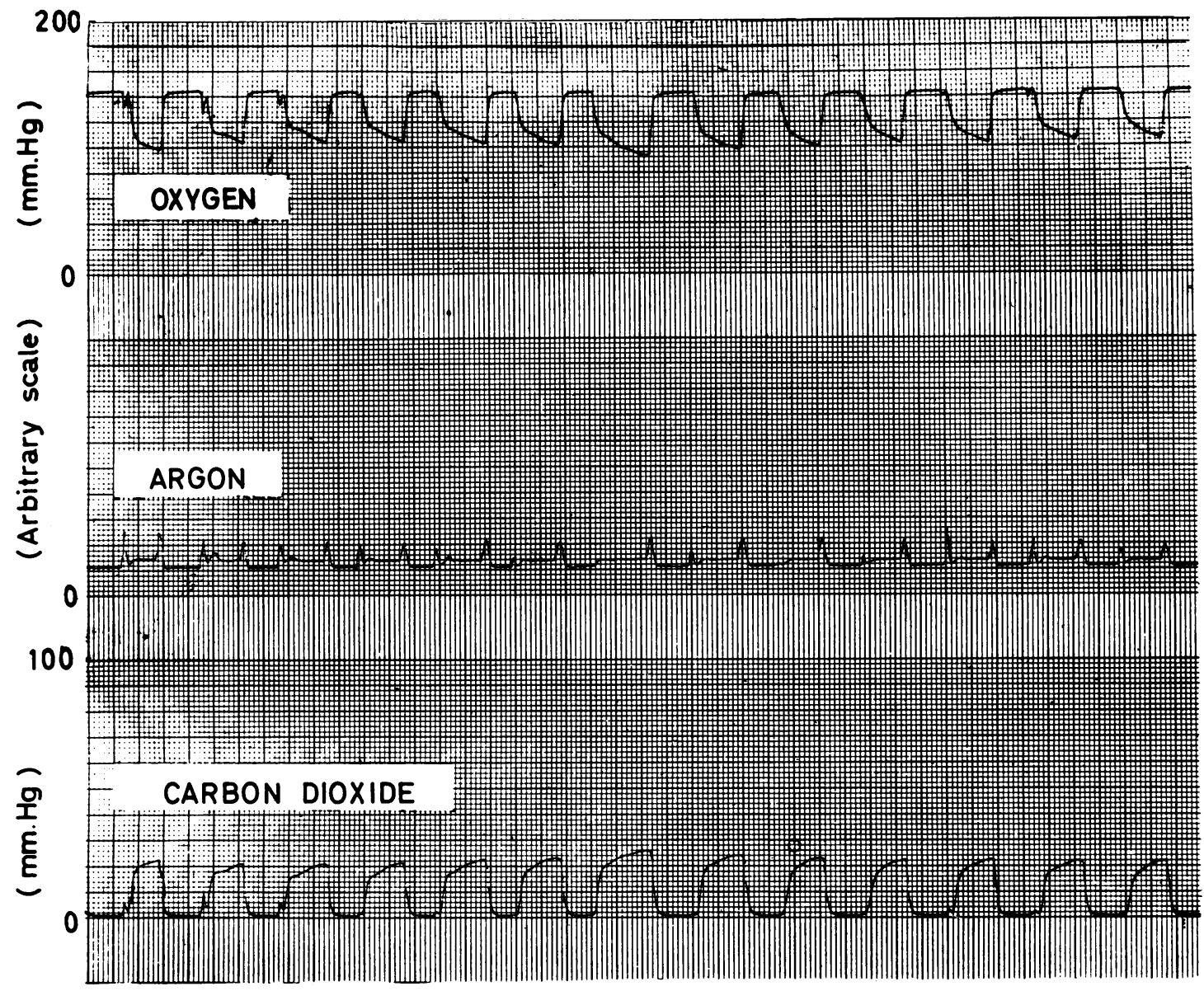

FIG. 11.-The pattern typical of mixed bronchial and extreme arterial obstruction artificially produced by labelling " pendelluft" gas with argon in a patient who only showed patterns characteristic of partial obstruction to ventilation. Time: 1 large square $=2$ seconds.

Although this " mixed" pattern is rare it is of great interest since the A and B pips can be used to obtain the instants of flow reversal and it is possible to derive information about some mechanical properties of the obstructed lobe if oesophageal pressure recordings are taken concurrently. The time constant of the pathological lobe can be calculated and the regional variations in airways resistance can be assessed (West and Hugh-Jones, 1959).

It was seen from the dog experiments above that the A "pendelluft" pip was often not very obvious in pure ventilatory obstruction but became more obvious in the presence of gross blood-flow obstruction because the "pendelluft" gas then had a different composition from the gas in the abnormal lobe. If the abnormal lobe has partial ventilatory obstruction but either a normal blood flow or one reduced proportionately to the ventilation, the "mixed" obstruction pattern is not produced. But it can artificially be produced by labelling the "pendelluft" gas from neighbouring areas of lung with argon or other convenient gas. An example of this technique is shown in Fig. 11, where the sampling tube was sited just proximal to a carcinoma partially obstructing the right main bronchus. Argon was trickled into the left main bronchus down another fine catheter. This technique of continuously trickling argon into the bronchial tree to label "pendelluft" gas can be used with oesophageal pressure measurements to estimate the mechanical properties of abnormal lobes and segments.

\section{Discussion}

The results presented show how, in qualitative terms, it is possible to assess changes in the venti- 
lation and blood flow in different lobes or segments of the lung during routine bronchoscopy. Other techniques, complementary to the observation of the changes in gas-concentration patterns, are now being developed which should enable more precise quantitative measurements of the ventilation, blood flow, and mechanical properties of the different individual lobes or segments to be made. Usually it is our present practice first to assess a patient by using oxygen- 15 , which enables the ventilation and blood flow in different zones of the lungs to be compared without any intubation of the patient (Dyson, Hugh-Jones, Newbery, and West, 1958; Dyson and others, 1960) and then, if required, bronchoscopy can be done and the anatomical localization of the variations in ventilation, blood flow, and mechanical changes into the relevant lobes or segments attempted. The two techniques of using short-lived gaseous isotopes and continuous gas analysis at bronchoscopy are thus conveniently complementary.

\section{SUMMARY}

The technique of continuous gas analysis from individual lobes and segments of the lungs during diagnostic bronchoscopy is described. This is achieved by inserting a sampling tube, from a special mass spectrometer, into the regional bronchus. The gas analysis is presented on a four-channel direct-writing recorder.
It is shown how simultaneous records of the local changes in oxygen, carbon dioxide, and argon (following introduction of argon into the bronchial tree) give characteristic patterns from which the presence of partial bronchial obstruction, arterial obstruction, or a combination of both in different lobes or segments can be inferred.

We wish to express our thanks to the Medical $\vec{\circ}$ Research Council for supporting this work; to Miss Heather MacLeish, Sisters N. M. Cooke and P. A. . Stock, and the nursing staff working in the Department $\overrightarrow{\overrightarrow{2}}$ of Medicine of the Postgraduate Medical School for $\vec{x}$ their help during the development of these techniques ; $\vec{O}$ and to Mr. Len Smith for his skill in constructing $i$ the various bronchoscopic sampling tubes which were $\vec{G}$ designed for use with the spectrometer.

\section{REFERENCE 3}

Dyson, N. A., Hugh-Jones, P., Newbery, G. R., and West, J. B. (1958). The Preparation and Use of Oxygen-15, with Particular $\subseteq$ Reference to its Value in the Study of Pulmonary Mal-function. ence on the Peaceful Uses of Atomic Energy. Vol. 26: Isotopes in Medicine, p. 103. Geneva.

1, 231. Sinclair, J. D., and West, J. B. (1960). Brit. med. J., ठூ

Fowler, K. T. (1957). A Mass Spectrometer for Rapid and Continuous Analysis of Gas Mixtures in Respiratory Research. Ph.D.O thesis. London University.

_ and Hugh-Jones, P. (1957). Brit. med. J., 1, 1205.

Macleod, W. M. (1954). Thorax, 9, 147.

Mattson, S. B., and Carlens, E. (1955). J. thorac. Surg., 30, 676.

Miller, R. D., Fowler, W. S., and Helmholz, H. F. (1956). J. Lab. (D clin. Med., 47, 297.

Svanberg, L. (1957). Scand. J. clin. Lab. Invest., 9, Suppl. 25.

West, J. B. (1960). J. appl. Physiol. In the press

— and Hugh-Jones, P. (1959). Ibid., 14, 743. Fowler, K. T., Hugh-Jones, P., and O'Donnell, T. V. (1957).
Clin. Sci., 16, 529 . 